\title{
Two-step method for precise calculation of core properties in molecules
}

\author{
A.V. Titov* N.S. Mosyagin, A.N. Petrov, and T.A. Isaev \\ Petersburg Nuclear Physics Institute, Gatchina, St.-Petersburg district 188300, Russia
}

\begin{abstract}
Precise calculations of core properties in heavy-atom systems which are described by the operators heavily concentrated in atomic cores, like to hyperfine structure and P,T-parity nonconservation effects, usually require accounting for relativistic effects. Unfortunately, completely relativistic treatment of molecules containing heavy elements is very consuming already at the stages of calculation and transformation of two-electron integrals with a basis set of four-component spinors.

In turn, the relativistic effective core potential (RECP) calculations of valence (spectroscopic, chemical etc.) properties of molecules are very popular because the RECP method allows one to treat quite satisfactory the correlation and relativistic effects for the valence electrons of a molecule and to reduce significantly the computational efforts. The valence molecular spinors are usually smoothed in atomic cores and, as a result, direct calculation of electronic densities near heavy nuclei is impossible.

In the paper, the methods of nonvariational and variational one-center restoration of correct shapes of four-component spinors in atomic cores after a two-component RECP calculation of a molecule are discussed. Their efficiency is illustrated in correlation calculations of hyperfine structure and parity nonconservation effects in heavy-atom molecules YbF, BaF, TIF, and PbO.
\end{abstract}

SHORT NAME: Studying core properties in molecules

KEYWORDS FOR INDEXING: electronic structure, atom in a molecule, molecules with heavy atoms, method of ab initio molecular calculation, relativistic effective core potential, one-center restoration.

\section{INTRODUCTION}

Historical background of study P-and T-parity nonconservation effects in heavy-atom molecules. After discovery of the combined charge (C) and space (P) parity violation, or CP-violation, in $K^{0}$-meson decay $[1]$, the search for the electric dipole moments (EDMs) of elementary particles has become one of the most fundamental problems in physics 2, 3, 4, 5. Permanent EDM is induced by the weak interaction that breaks both the space symmetry inversion and time-reversal invariance $(\mathrm{T})[6]$. Considerable experimental effort has been invested in measuring atomic EDMs induced by the EDMs of proton, neutron and electron and by P,T-odd interactions between them. The best available restriction for the electron EDM, $d_{e}$, was obtained in the atomic Tl experiment [7], which established an upper limit of $\left|d_{e}\right|<1.6 \times 10^{-27} e \cdot \mathrm{cm}$, where $e$ is the charge of the electron. The benchmark upper limit on a nucleus EDM is obtained in atomic experiment on ${ }^{199} \mathrm{Hg}[8],\left|d_{\mathrm{Hg}}\right|<2.1 \times 10^{-28} e \cdot \mathrm{cm}$, from which the best restriction on the proton EDM, $\left|d_{p}\right|<5.4 \times 10^{-24} e \cdot \mathrm{cm}$, was also recently obtained by Dmitriev \& Sen'kov $[9]$.

Since 1967, when Sandars suggested to use polar heavy-atom molecules in the experimental search for the proton EDM 10], the molecules are considered as the most promising objects for such experiments. Sandars also noticed earlier 11] that the P- and P,T-parity nonconservation (PNC) effects are strongly enhanced in heavy-atom systems due to relativistic and other effects. For example, in paramagnetic atoms the enhancement factor for an electron

*Electronic address: titov@pnpi.spb.ru URL: http://www.qchem.pnpi.spb.ru 
$\mathrm{EDM}, d_{\text {atom }} / d_{e}$, is roughly proportional to $\alpha^{2} Z^{3} \alpha_{D}$, where $\alpha \approx 1 / 137$ is the fine structure constant, $Z$ is the nuclear charge and $\alpha_{D}$ is the atomic polarisability. It can be of order 100 or greater for highly polarizable heavy atoms $(Z \geq 50)$. Furthermore, the effective inner molecular electric field $E_{\text {mol }}$ acting on electrons in polar molecules can be a few orders of magnitude higher than the maximal field $E_{\text {ext }}$ accessible in a laboratory, $E_{\mathrm{mol}} / E_{\text {ext }} \sim 10^{5}$. The first molecular EDM experiment (on search for the proton EDM and other nuclear P,T-odd effects) was performed on TIF by Sandars et al. 12] (Oxford, UK). In 1991, in the last series of the ${ }^{205} \mathrm{TlF}$ experiments by Hinds et al. 13] (Yale, USA), the restriction $d_{p}=(-4 \pm 6) \times 10^{-23} e \cdot \mathrm{cm}$ was obtained (that was recalculated in 2002 by Petrov et al. [14] as $\left.d_{p}=(-1.7 \pm 2.8) \times 10^{-23} e \cdot \mathrm{cm}\right)$.

In 1978 the experimental investigation of the electron EDM and other PNC effects was further stimulated by Labzowsky et al. 15, 16] and Sushkov \& Flambaum [17] who clarified the possibilities of additional enhancement of these effects in diatomic radicals like $\mathrm{BiS}$ and $\mathrm{PbF}$ due to the closeness of levels of opposite parity in $\Omega$-doublets having the ${ }^{2} \Pi_{1 / 2}$ ground state. Then Sushkov et al. [18] and Flambaum \& Khriplovich [19] have suggested to use $\Omega$ doubling in diatomic radicals with the ${ }^{2} \Sigma_{1 / 2}$ ground state for such experiments and the $\mathrm{HgF}, \mathrm{HgH}$ and BaF molecules were first studied semiempirically by Kozlov [20]. At the same time, the first two-step ab initio calculation of PNC effects in PbF initiated by Labzowsky was finished in St.-Petersburg (Russia) 21]. A few years later, Hinds started experimental search for the electron EDM on the YbF molecule, on which the first result was obtained by his group in 2002 (Sussex, UK) 22], $d_{e}=(-0.2 \pm 3.2) \times 10^{-26} e \cdot \mathrm{cm}$. Though that restriction is worse than the best available now $d_{e}$ datum calculated from the $\mathrm{Tl}$ experiment (see above), nevertheless, it is limited by only counting statistics, as Hinds et al. pointed out in 22$]$.

New series of the electron EDM experiments on YbF by Hinds group (London, UK) are in progress and new generation of the electron EDM experiments using a vapor cell, on the metastable $a(1)$ state of $\mathrm{PbO}$, is prepared by group of DeMille (Yale, USA). The unique suitability of $\mathrm{PbO}$ for searching the elusive $d_{e}$ is demonstrated by the very high statistical sensitivity of the Yale experiment to the electron EDM. In prospect, it allows one to detect $d_{e}$ of order of $10^{-31} e \cdot \mathrm{cm}$ [23], three-four orders of magnitude lower than the current limit quoted above. Some other candidates for the EDM experiments, $\mathrm{HgH}, \mathrm{HgF}$, and $\mathrm{TeO}^{*}$, are yet discussed and the experiment on $\mathrm{PbF}$ is planned in Oklahoma, USA.

In order to interpret the data measured in such molecular experiments in terms of the electron EDM or other fundamental constants of P- and P,T-odd interactions, high-precision calculation of the electronic wave function near a heavy nucleus is required. Moreover, ab initio calculations of some molecular properties are usually required already prior to the stage of preparation of the experimental setup.

Heavy-atom molecules, computational strategies. The most straightforward way for calculation of electronic structure of molecules containing heavy atoms is solution of the eigenvalue problem using the Dirac-Coulomb or DiracCoulomb-Breit Hamiltonian [24, 25, 26] when some approximation for the four-component wave function is chosen.

However, even applying the relativistic SCF approximation to heavy-atom molecules when solving the Dirac-Fock or Dirac-Fock-Breit equations followed by the transformation of the two-electron integrals is not always an easy task because much larger sets of primitive basis functions are required [27, 28] for such all-electron four-component calculations as compared to the nonrelativistic case. Starting from the Pauli approximation and Foldy \& Wouthuysen transformation, many different two-component approaches were developed in which only large components are treated explicitly (e.g., see [29, 30, 31, 32] and references).

The most computational savings are yet achieved when the two-component relativistic effective core potential (RECP) approximation proposed by Lee et al. 33] is used [34, 35]. There are the following reasons for it. The RECP approach allows one to exclude large number of chemically inactive electrons from molecular calculations and to treat explicitly the valence electrons only. Then, the oscillations of the valence spinors are usually smoothed in heavy-atom 
cores. As a result, the number of primitive basis functions can be reduced dramatically. This approach is based on a well-developed nonrelativistic technique of calculations, however, an effective spin-orbit interaction and other scalarrelativistic effects are taken into account usually by means of radially-local or separable potentials [34, 36]. Many complications of the Dirac-Coulomb(-Breit) molecular calculations [37] are avoided when employing RECPs. The radially-local RECP approaches for "shape-consistent" (or "norm-conserving") pseudoorbitals are the most widely applied in calculations of molecules with heavy elements though "energy-adjusted/consistent" pseudopotentials [35] by Stuttgart team and Huzinaga-type "ab initio model potentials" [38] are also actively used. (In plane wave calculations of many-atomic systems and in molecular dynamics, the separable pseudopotentials [36, 39, 40, 41, 42] are more popular now because they provide linear scaling of computational effort on the basis set size.) The nonrelativistic shape-consistent effective core potential has been first proposed by Durand \& Barthelat [43] and then a modified scheme of the pseudoorbital constructing was suggested by Christiansen et al. [44] and by Hamann et al. [45].

However, inaccuracy of the conventional radially-local RECP approaches reaches 1000-3000 $\mathrm{cm}^{-1}$ for the transition and dissociation energies that may be insufficient in practice. In deep reorganization of electronic configuration structure of molecules containing, in particular, transition metals, lanthanides, actinides, and superheavy elements the conventional radially-local RECP approaches can not provide reliable results for a wide variety of properties (as it was shown both in many calculations and theoretically, see [46, 47] and references) though otherwise is sometime stated (e.g., see [48, 49]). Such problems can be efficiently overcomed by applying the generalized RECP (GRECP) approach [46, 47, 50, 51, 52], that involves both radially-local, separable and Huzinaga-type [38, 53, 54] potentials as its components and as particular cases. In the GRECP concept, the inner core, outer core and valence electrons are first treated employing different approximations for each.

Nevertheless, calculation of such properties as electronic densities near nuclei, hyperfine structure constants, P,Tparity nonconservation effects, chemical and isotopic shifts etc. with the help of the two-component pseudospinors smoothed in cores is impossible. For evaluation the matrix elements of the operators singular on nuclei, proper shapes of the valence molecular four-component spinors must be restored in atomic core regions after the RECP calculation of that molecule performed.

In 1959, a nonrelativistic procedure of restoration of the orbitals from smoothed Phillips-Kleinman pseudoorbitals was proposed $55 \mid$ based on the orthogonalization of the latters to the original atomic core orbitals. In 1985 , Pacios \& Christiansen [56] suggested a modified orthogonalization scheme in the case of shape-consistent pseudospinors. At the same time, a simple procedure of one-center restoration employing the idea of generation of equivalent basis sets in four-component Dirac-Fock and two-component RECP/SCF calculations was proposed in [21] (i.e. NOCR procedure, see below) and first applied to evaluation of the P,T-odd spin-rotational Hamiltonian parameters in the PbF molecule. In 1994, similar procedure was used by Blöchl inside the augmentation regions [57] in solids to construct the transformation operator between pseudoorbitals ("PS") and original orbitals ("AE") in his projector augmented-wave method.

All the above restoration schemes can be called by "nonvariational" as compared to the "variational" one-center restoration (VOCR, see below) procedure proposed in [58, 59]. Proper behavior of the molecular orbitals (fourcomponent spinors in the relativistic case) in atomic cores of molecules can be restored in the scope of a variational procedure if the molecular pseudoorbitals (two-component pseudospinors) match correctly the original orbitals (large components of bispinors) in the valence region after the molecular RECP calculation. This condition is rather correct when the shape-consistent RECP is involved to the molecular calculation with explicitly treated outermost core orbitals and, especially, when the GRECP operator is used as is demonstrated in [46].

At the restoration stage, a one-center expansion on the spherical harmonics with numerical radial parts is most appropriate both for orbitals (spinors) and for the description of "external" interactions with respect to the core regions 
of a considered molecule. In the scope of the discussed two-step methods of the electronic structure calculation of a molecule, finite nucleus models and quantum electrodynamic terms including, in particular, two-electron Breit interaction [51] may be used without problems.

One-center expansion had been applied first to whole molecules by Desclaux \& Pyykkö in relativistic and nonrelativistic Hartree-Fock calculations for the series $\mathrm{CH}_{4}$ to $\mathrm{PbH}_{4}[60$ ] and then in the Dirac-Fock calculations of CuH, $\mathrm{AgH}$ and $\mathrm{AuH}$ 61] and other molecules 62]. A large bond length contraction due to the relativistic effects has been estimated. However, the accuracy of such calculations is limited in practice because the orbitals of the hydrogen atom are reexpanded on a heavy nucleus in all the coordinate space. It is worth to note that the RECP and one-center expansion approaches were considered earlier as alternatives to each other [63, 64].

The applicability of the proposed two-step algorithms for calculation of wave functions of molecules with heavy atoms is a consequence of the fact that the valence and core electrons may be considered as two subsystems, interaction between which is described mainly by some integrated and not by detailed properties of these subsystems. The methods for consequent calculation of the valence and core parts of electronic structure of molecules give us a way to combine relative simplicity and accessibility of both molecular RECP calculations in gaussian basis set and relativistic finite-difference one-center calculations inside a sphere with the atomic core radius.

The first two-step calculations of the P,T-odd spin-rotational Hamiltonian parameters were performed for the $\mathrm{PbF}$ radical about 20 years ago [21, 65] with the semiempirical accounting for the spin-orbit interaction. Before, only nonrelativistic SCF calculation of the TlF molecule using the relativistic scaling was carried out [66, 67], in which the P,T-odd values were almost three times underestimated as compared to the relativistic DF calculations. The latter were first performed only in 1997 by Laerdahl et al. 68] and by Parpia [69]. The next two-step calculation, for $\mathrm{PbF}$ and $\mathrm{HgF}$ molecules 70 , was carried out with the spin-orbit RECP part taken into account using the method suggested in [71].

Later we performed correlation GRECP/NOCR calculations of the core properties in YbF [2], BaF [3], again in YbF 74] and in TIF [14]. In 1998, first all-electron Dirac-Fock calculations of the YbF molecule were also performed by Quiney et al. [75] and by Parpia 76]. Very recently we finished extensive two-step calculations of the P,T-odd properties and hyperfine structure of the excited states of the $\mathrm{PbO}$ molecule [77, 78].

In the paper, the main features of the used two-step method are presented and only the last series of the twostep calculations are discussed, in which electron correlations are taken into account by a combined method of the second-order perturbation theory (PT2) and configuration interaction (CI), or "PT2/CI" 79] (for BaF and YbF), by the relativistic coupled cluster (RCC) method 80, 81] (for TlF and PbO), and by the spin-orbit direct-CI method 82, 83, 84] (for $\mathrm{PbO}$ ). In the discussed ab initio calculations the best up to-date accuracy was attained for the hyperfine constants and P,T-odd parameters regarding the molecules containing heavy atoms.

\section{GENERALIZED RELATIVISTIC EFFECTIVE CORE POTENTIAL METHOD.}

When core electrons of a heavy-atom molecule do not play an active role, the effective Hamiltonian with RECP can be presented in the form

$$
\mathbf{H}^{\mathrm{Ef}}=\sum_{i_{v}}\left[\mathbf{h}^{\mathrm{Schr}}\left(i_{v}\right)+\mathbf{U}^{\mathrm{Ef}}\left(i_{v}\right)\right]+\sum_{i_{v}>j_{v}} \frac{1}{r_{i_{v} j_{v}}} .
$$

Hamiltonian (1) is written only for a valence subspace of electrons which are treated explicitly and denoted by indices $i_{v}$ and $j_{v}$. In practice, this subspace is often extended by inclusion of some outermost core shells for better accuracy but we will consider them as the valence shells below if these outermost core and valence shells are not treated 
using different approximations. $\mathbf{U}^{\mathrm{Ef}}$ is an RECP (or relativistic pseudopotential) operator that is usually written in the separable (e.g., see [36] and references) or radially-local (semi-local) 34] approximations when the valence pseudospinors are smoothed in the heavy-atom cores. The RECP operator simulates, in particular, interactions of the explicitly treated electrons with those which are excluded from the RECP calculations. Besides, the generalized RECP operator [46, 50] described below can be used that includes the radially-local, separable and Huzinaga-type relativistic pseudopotentials as special cases. Additionally, the GRECP operator can include terms of other types which are important first of all for economical but precise treatment of transition metals, lanthanides and actinides. With these terms, accuracy provided by GRECPs can be even higher than the accuracy of the frozen core approximation employing the same separation on subspaces of valence and core electrons [46, 47]. In Eq. (11), $\mathbf{h}^{\text {Schr }}$ is the one-electron Schrödinger Hamiltonian

$$
\mathbf{h}^{\mathrm{Schr}}=-\frac{1}{2} \vec{\nabla}^{2}-\frac{Z_{i c}}{r},
$$

where $Z_{i c}$ is the charge of the nucleus decreased by the number of inner core electrons. Contrary to the four-component wave function used in Dirac-Coulomb(-Breit) calculations, the pseudo-wave function in the (G)RECP case can be both two- and one-component. The use of the effective Hamiltonian (1) instead of the all-electron relativistic Hamiltonians leads to the question about its accuracy. It was shown both theoretically and in many calculations (see [6] and references) that the usual accuracy of the radially-local RECP versions is within $1000-3000 \mathrm{~cm}^{-1}$ for transition energies between low-lying states.

The GRECP concept is introduced and developed in a series of papers (see [46, 50, 52] and references). In contrast to other RECP methods, GRECP employs the idea of separating the space around a heavy atom into three regions: inner core, outer core and valence, which are treated differently. It allows one to attain practically any desired accuracy, while requiring moderate computational efforts.

The main steps of the scheme of generating the GRECP version with the separable correction taken into account are:

1. The numerical all-electron relativistic calculation of a generator state is carried out for an atom under consideration. For this purpose, we use the atomic HFDB code [85, 86].

2. The numerical pseudospinors $\tilde{f}_{n l j}(r)$ are constructed of the large components $f_{n l j}(r)$ of the outer core (OC) and valence (V) $\operatorname{HFD}(\mathrm{B})$ spinors so that the innermost pseudospinors of them (for each $l$ and $j$ ) are nodeless, the next pseudospinors have one node, and so forth. These pseudospinors satisfy the following conditions:

$$
\begin{gathered}
\tilde{f}_{n l j}(r)= \begin{cases}f_{n l j}(r), & r \geq R_{c}, \\
y(r)=r^{\gamma} \sum_{i=0}^{5} a_{i} r^{i}, & r<R_{c},\end{cases} \\
l=0,1, \ldots, L, \quad j=\left|l \pm \frac{1}{2}\right|, \\
n=n_{o c}, n_{o c}^{\prime}, \ldots, n_{v},
\end{gathered}
$$

where $n_{v}, n_{o c}, n_{o c}^{\prime}$ are principal quantum numbers of the valence and outer core spinors, $L$ is one more than the highest orbital angular momentum of the inner core (IC) spinors. The leading power $\gamma$ in the polynomial is typically chosen to be close to $L+1$ in order to ensure sufficient ejection of the valence and outer core electrons from the inner core region. The $a_{i}$ coefficients are determined by the following requirements:

- $\left\{\tilde{f}_{n l j}\right\}$ set is orthonormalized,

- $y$ and its first four derivatives match $f_{n l j}$ and its derivatives, 
- $y$ is a smooth and nodeless function, and

- $\tilde{f}_{n l j}$ ensures a sufficiently smooth shape of the corresponding potential.

$R_{c}$ is chosen near the extremum of the spinor so that the corresponding pseudospinor has the defined above number of nodes. In practice, the $R_{c}$ radii for the different spinors should be chosen close to each other to generate smooth potentials.

3. The $U_{n l j}$ potentials are derived for each $l=0, \ldots, L$ and $j=\left|l \pm \frac{1}{2}\right|$ for the valence and outer core pseudospinors so that the $\tilde{f}_{n l j}$ are solutions of the nonrelativistic-type Hartree-Fock equations in the $j$-coupling scheme for a "pseudoatom" with the removed inner core electrons.

$$
\begin{aligned}
& U_{n l j}(r)=\tilde{f}_{n l j}^{-1}(r)\left[\left(\frac{1}{2} \frac{\mathbf{d}^{2}}{\mathbf{d r}^{\mathbf{2}}}-\frac{l(l+1)}{2 r^{2}}+\frac{Z_{i c}}{r}-\widetilde{\mathbf{J}}(r)+\widetilde{\mathbf{K}}(r)+\varepsilon_{n l j}\right) \tilde{f}_{n l j}(r)+\right. \\
& \left.\sum_{n^{\prime} \neq n} \widetilde{\varepsilon}_{n^{\prime} n l j} \tilde{f}_{n^{\prime} l j}(r)\right],
\end{aligned}
$$

where $\widetilde{\mathbf{J}}$ and $\widetilde{\mathbf{K}}$ are the Coulomb and exchange operators calculated with the $\tilde{f}_{n l j}$ pseudospinors, $\varepsilon_{n l j}$ are the oneelectron energies of the corresponding spinors, and $\widetilde{\varepsilon}_{n^{\prime} n l j}$ are off-diagonal Lagrange multipliers (which are, in general, slightly different for the original bispinors and pseudospinors).

4. The GRECP operator with the separable correction written in the spinor representation [46] is as

$$
\begin{aligned}
\mathbf{U}^{\mathrm{Ef}} & =U_{n_{v} L J}(r)+\sum_{l=0}^{L} \sum_{j=|l-1 / 2|}^{l+1 / 2}\left\{\left[U_{n_{v} l j}(r)-U_{n_{v} L J}(r)\right] \mathbf{P}_{l j}\right. \\
& +\sum_{n_{o c}}\left[U_{n_{o c}, l j}(r)-U_{n_{v} l j}(r)\right] \widetilde{\mathbf{P}}_{n_{o c}, l j}+\sum_{n_{o c}} \widetilde{\mathbf{P}}_{n_{o c}, l j}\left[U_{n_{o c}, l j}(r)-U_{n_{v} l j}(r)\right] \\
& \left.-\sum_{n_{o c}, n_{o c}^{\prime}} \widetilde{\mathbf{P}}_{n_{o c}, l j}\left[\frac{U_{n_{o c}, l j}(r)+U_{n_{o c}^{\prime}, l j}(r)}{2}-U_{n_{v} l j}(r)\right] \widetilde{\mathbf{P}}_{n_{o c}^{\prime}, l j}\right\},
\end{aligned}
$$

where

$$
\mathbf{P}_{l j}=\sum_{m=-j}^{j}|l j m\rangle\left\langle l j m\left|, \quad \widetilde{\mathbf{P}}_{n_{o c}, l j}=\sum_{m=-j}^{j}\right| \widetilde{n_{o c}, l j} m\right\rangle\left\langle n_{o c}, \widetilde{l j} m\right|,
$$

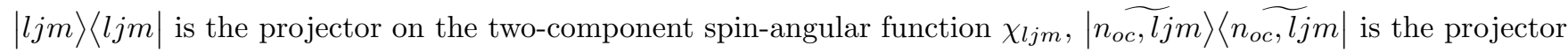
on the outer core pseudospinors $\tilde{f}_{n_{o c}, l j} \chi_{l j m}$, and $J=L+1 / 2$.

Two of the major features of the GRECP version with the separable correction described here are generating of the effective potential components for the pseudospinors which may have nodes, and addition of non-local separable terms with projectors on the outer core pseudospinors (the second and third lines in Eq. (515) to the standard semilocal RECP operator (the first line in Eq. (5)). Some other GRECP versions are described and discussed in papers 46, 47, 50, 52 in details.

The GRECP operator in spinor representation (5) is mainly used in our atomic calculations. The spin-orbit representation of this operator which can be found in [46] is more efficient in practice being applied to molecular calculations. Despite the complexity of expression (5) for the GRECP operator, the calculation of its one-electron integrals is not notably more expensive than that for the case of the conventional radially-local RECP operator. 


\section{NONVARIATIONAL ONE-CENTER RESTORATION METHOD}

The electronic density evaluated from the two-component pseudo-wave function (that, in turn, is obtained in the (G)RECP calculation accounting for correlation) very accurately reproduces the corresponding all-electron fourcomponent density in the valence and outer core regions. In the inner core region, the pseudospinors are smoothed, so that the electronic density with the pseudo-wave function is not correct. When operators describing properties of interest are heavily concentrated near the nucleus, their mean values are strongly affected by the wave function in the inner region. The four-component molecular spinors must, therefore, be restored in the heavy-atom cores.

All molecular spinors $\phi_{p}$ can be restored as one-center expansions in the cores using the nonvariational one-center restoration (NOCR) scheme [21, 65, 70, 72, 73, 74, 14, 77, 78] that consists of the following steps:

- Generation of equivalent basis sets of one-center four-component spinors $\left(\begin{array}{c}f_{n l j}(r) \chi_{l j m} \\ g_{n l j}(r) \chi_{2 j-l, j m}\end{array}\right)$ and of smoothed two-component pseudospinors $\tilde{f}_{n l j}(r) \chi_{l j m}$ in finite-difference all-electron Dirac-Fock(-Breit) and GRECP/SCF calculations of the same configurations of a considered atom and its ions. The nucleus is usually modeled as a uniform charge distribution within a sphere. The all-electron four-component HFDB [85, 86, 87] and two-component GRECP/HFJ [88, 89] codes are employed by us to generate two equivalent numerical basis sets used at the restoration. These sets, describing mainly the atomic core region, are generated independently of the basis set used for the molecular GRECP calculations.

- The molecular pseudospinorbitals are then expanded in the basis set of one-center two-component atomic pseudospinors (for $r \leq R_{\text {nocr }}$, where $R_{\text {nocr }}$ is the radius of restoration that should be sufficiently large for calculating core properties with required accuracy),

$$
\tilde{\phi}_{p}(\mathbf{x}) \approx \sum_{l=0}^{L_{\text {max }}} \sum_{j=|l-1 / 2|}^{j=|l+1 / 2|} \sum_{n, m} c_{n l j m}^{p} \tilde{f}_{n l j}(r) \chi_{l j m},
$$

where $\mathbf{x}$ denotes spatial and spin variables. Note that for linear molecules only one value of $m$ survives in the sum for each $\phi_{p}$.

- Finally, the atomic two-component pseudospinors in the molecular basis are replaced by equivalent four-component spinors and the expansion coefficients from Eq. (6) are preserved:

$$
\phi_{p}(\mathbf{x}) \approx \sum_{l=0}^{L_{\max }} \sum_{j=|l-1 / 2|}^{j=|l+1 / 2|} \sum_{n, m} c_{n l j m}^{p}\left(\begin{array}{c}
f_{n l j}(r) \chi_{l j m} \\
g_{n l j}(r) \chi_{2 j-l, j m}
\end{array}\right) .
$$

The molecular four-component spinors constructed this way are orthogonal to the inner core spinors of the heavy atom, because the atomic basis functions used in Eq. (7) are generated with the inner core electrons treated as frozen.

\section{VARIATIONAL ONE-CENTER RESTORATION}

In the variational technique of the one-center restoration (VOCR) 58, 59], the proper behavior of the fourcomponent molecular spinors in the core regions of heavy atoms can be restored as an expansion on the spherical harmonics inside the sphere with a restoration radius, $R_{\mathrm{vocr}}$, that should not be smaller than the matching radius, $R_{c}$, used at the RECP generation. The outer parts of spinors are treated as frozen after the RECP calculation of a considered molecule. This method enables one to combine the advantages of two well-developed approaches, molecular 
RECP calculation on gaussian basis set and atomic-type one-center calculation on numerical basis functions, by the most optimal way. It is considered theoretically in [59] and some results concerning the efficiency of the one-center reexpansion of orbitals on another atom can be found in [47].

The VOCR scheme can be used for constructing the core parts of the molecular spinors (orbitals) instead of using equivalent basis sets as is in the NOCR technique. Some disadvantage of the NOCR scheme is that molecular pseudoorbitals are usually computed in a spin-averaged GRECP/SCF molecular calculation (i.e. without accounting for the effective spin-orbit interaction) and the reexpansion of molecular pseudospinorbitals on one-center atomic pseudospinors is yet restricted by accuracy, as it was seen in our GRECP/RCC/NOCR calculations 14] of the TIF molecule (see below). With the restored molecular bispinors, the two-electron integrals on molecular bispinors can be easily calculated when following the scheme presented in Eqs. (9)-(12) of the next section (see [59] for more details). Thus, the four-component transfomation from the atomic basis that is now the most time-consuming stage of four-component calculations of heavy-atom molecules can be avoided.

However, the most interesting direction in development of the two-step method is the possibility to "split" the correlation structure calculation of a molecule on two consequent correlation calculations: first, in the valence region, when the outer core and valence electrons are explicitly involved into the GRECP calculation, and then, in the core region, when the outer and inner core space regions are only treated at the restoration stage. Correlation effects in the valence and outer core regions (not only valence parts of molecular orbitals but also configuration coefficients) can be calculated, for example, by the GRECP/RCC method with very high accuracy. Then correlation effects in the inner and outer core regions can be taken into account using the Dirac-Coulomb(-Breit) Hamiltonian and the one-center expansion. Increasing the one-center restoration radius $R_{\mathrm{vocr}}$ one can take into account correlation effects in the intermediate region (outer core in our case) with the required accuracy. Roughly speaking, the computational efforts for the correlation structure calculations in the core and valence regions are added when using the two-step approach, whereas in the conventional one-step scheme, they have multiplicative nature.

\section{TWO-STEP CALCULATION OF MOLECULAR PROPERTIES.}

To evaluate one-electron core properties (hyperfine structure, P,T-odd effects etc.) employing the above restoraton schemes it is sufficient to obtain the one-particle density matrix, $\left\{\widetilde{D_{p q}}\right\}$, after the molecular RECP calculation on the basis of pseudospinors $\left\{\widetilde{\phi}_{p}\right\}$. At the same time, the matrix elements $\left\{W_{p q}\right\}$ of the property operator $\boldsymbol{W}(\mathbf{x})$ should be calculated on the basis of equivalent four-component spinors $\left\{\phi_{p}\right\}$. The mean value for this operator can be then evaluated as

$$
\langle\boldsymbol{W}\rangle=\sum_{p q} \widetilde{D_{p q}} W_{p q}
$$

The only explicitly treated valence shells are used for evaluating a core property when applying Eq. (8) since the atomic frozen core (closed) shells do not usually contibute to the properties of practical interest. However, correlations with the core electrons explicitly excluded from the RECP calculation can be also taken into account if the effective operator technique [90] is applied to calculate the property matrix elements $\left\{W_{p q}^{\mathrm{Ef}}\right\}$ on the basis set of bispinors $\left\{\phi_{p}\right\}$. Then, in expression (8) one should only replace $\left\{W_{p q}\right\}$ by $\left\{W_{p q}^{\text {Ef }}\right\}$. Alternatively, the correlations with the inner core electrons can be, in principle, considered within the variational restoration scheme for electronic structure in the heavy atom cores. Strictly speaking, the use of the effective operators is correct when the molecular calculation is carried out with the "correlated" GRECP (see [52]), in which the same correlations with the excluded core electrons are taken into account at the GRECP generation as they are in constructing $\left\{W_{p q}^{\mathrm{Ef}}\right\}$. Nevertheless, the use of the $(\mathrm{G}) \mathrm{RECP}$ that 
does not account for the core correlations at the molecular calculation stage can be justified if they do not influence dramatically on the electronic structure in the valence region. The latter approximation was applied in calculations of the $\mathrm{YbF}$ and $\mathrm{BaF}$ molecules described in the following section.

When contributions to $\langle\boldsymbol{W}\rangle$ are important both in the core and valence regions, the scheme for evaluating the mean value of $\boldsymbol{W}(\mathbf{x})$ can be as follows:

- calculation of matrix elements on the molecular pseudospinorbitals (which are usually linear combination of atomic gaussians) over all the space region using conventional codes for molecular RECP calculations (though, the operator $\boldsymbol{W}$ can be presented in different forms in calculations with the RECP and Dirac-Coulomb(-Breit) Hamiltonians),

$$
\widetilde{\langle\boldsymbol{W}\rangle}=\sum_{p q} \widetilde{D_{p q}} \int_{r<\infty} \widetilde{\phi}_{p}(\mathbf{x}) \boldsymbol{W}(\mathbf{x}) \widetilde{\phi}_{q}(\mathbf{x}) d \mathbf{x} ;
$$

- calculation of the inner part of the matrix element of the operator with the same molecular pseudospinorbitals using the one-center expansion for $\left\{\widetilde{\phi}_{p}\right\}\left(R_{\text {ocr }}\right.$ stays for $R_{\text {nocr }}$ or $R_{\text {vocr }}$ below, $\left.R_{\text {ocr }} \geq R_{c}\right)$ :

$$
\widetilde{\langle\boldsymbol{W}\rangle}<=\sum_{p q} \widetilde{D_{p q}} \int_{r<R_{\mathrm{ocr}}} \widetilde{\phi}_{p}(\mathbf{x}) \boldsymbol{W}(\mathbf{x}) \widetilde{\phi}_{q}(\mathbf{x}) d \mathbf{x} ;
$$

- calculation of the inner part of the matrix element of the operator with the restored molecular four-component spinors using the one-center expansion for $\left\{\phi_{p}\right\}$ :

$$
\langle\boldsymbol{W}\rangle^{<}=\sum_{p q} \widetilde{D_{p q}} \int_{r<R_{\text {ocr }}} \phi_{p}^{<}(\mathbf{x}) \mathbf{W}(\mathbf{x}) \phi_{\mathbf{q}}^{<}(\mathbf{x}) \mathbf{d x} .
$$

The matrix element $\langle\boldsymbol{W}\rangle$ of the $\boldsymbol{W}(\mathbf{x})$ operator is evaluated as

$$
\langle\boldsymbol{W}\rangle=\widetilde{\langle\boldsymbol{W}\rangle}-\widetilde{\langle\boldsymbol{W}}\rangle^{<}+\langle\boldsymbol{W}\rangle^{<} .
$$

Obviously, that the one-center basis functions can be numerical (finite-difference) for better flexibility.

The mean values of the majority of operators for the valence properties can be calculated with good accuracy without accounting for the inner parts of the matrix elements (10) and (11). As is discussed above, for calculating the mean values of the operators singular near nuclei it is sufficient to account only for the inner parts, (11), of the matrix elements of $\boldsymbol{W}(\mathbf{x})$ on the restored functions $\phi_{p}^{<}(\mathbf{x})$, whereas the other contributions, (9) and (10), can be neglected.

Calculation of properties using the finite-field method [91, 92] and Eq. (8) within the coupled-cluster approach is described in section DT

\section{CALCULATION OF SPIN-ROTATIONAL HAMILTONIAN PARAMETERS FOR YBF AND BAF MOLECULES}

Effective Hamiltonian. The molecular spin-rotational degrees of freedom of $\mathrm{YbF}$ and $\mathrm{BaF}$ for the ${ }^{171} \mathrm{Yb}$ and ${ }^{137} \mathrm{Ba}$ isotopes with nuclear spin $I=\frac{1}{2}$ are described by Hamiltonian 65 , 70, 93 ]

$$
\begin{aligned}
\mathbf{H}_{\mathrm{sr}} & =B \overrightarrow{\mathbf{N}}^{2}+\gamma \overrightarrow{\mathbf{S}} \overrightarrow{\mathbf{N}}-D_{e} \vec{\lambda} \vec{E}+\overrightarrow{\mathbf{S}} \hat{\mathbf{A}} \overrightarrow{\mathbf{I}} \\
& +W_{\mathrm{A}} k_{\mathrm{A}} \vec{\lambda} \times \mathbf{\mathbf { S }} \cdot \overrightarrow{\mathbf{I}}+\left(W_{\mathrm{S}} k_{\mathrm{S}}+W_{d} d_{\mathrm{e}}\right) \overrightarrow{\mathbf{S}} \vec{\lambda}
\end{aligned}
$$

where $\overrightarrow{\mathbf{N}}$ is the rotational angular momentum, $B$ is the rotational constant, $\overrightarrow{\mathbf{S}}$ is the effective spin of the electron 93], $\overrightarrow{\mathbf{I}}$ is the spin of the ${ }^{171} \mathrm{Yb}\left({ }^{137} \mathrm{Ba}\right)$ nucleus, $\vec{\lambda}$ is the unit vector directed along the molecular axis from the heavy 
nucleus to fluorine. The spin-doubling constant $\gamma$ characterizes the spin-rotational interaction. $D_{e}$ and $\vec{E}$ are the molecular dipole moment and the external electric field. The axial tensor $\hat{\mathbf{A}}$ describes magnetic hyperfine structure on the ytterbium (barium) nucleus:

$$
\mathbf{H}_{\mathrm{hfs}}=\frac{\mu_{N}}{I} \frac{\overrightarrow{\mathbf{I}} \cdot \vec{\alpha} \times \vec{r}}{r^{3}}, \quad \vec{\alpha}=\left(\begin{array}{cc}
0 & \vec{\sigma} \\
\vec{\sigma} & 0
\end{array}\right),
$$

which can be also expressed in the notations of the isotropic $A=\left(A_{\|}+2 A_{\perp}\right) / 3$ and dipole $A_{\mathrm{d}}=\left(A_{\|}-A_{\perp}\right) / 3$ hyperfine structure constants. The hyperfine interaction with the ${ }^{19} \mathrm{~F}$ nucleus is significantly smaller and is not of interest for the considered effects.

The last three terms in Eq. (13) account for the P- and P,T-odd effects: the first of them describes the electromagnetic interaction of the electron with the anapole moment of the nucleus with the constant $k_{\mathrm{A}}[94$; the second term accounts for the scalar P,T-odd electron-nucleon interaction characterized by the dimensionless constant $k_{\mathrm{S}}$; the third term corresponds to interaction of the electron EDM $d_{\mathrm{e}}$ with the internal molecular field $\vec{E}_{\mathrm{mol}}$ :

$$
\begin{aligned}
& \mathbf{H}_{d}=2 d_{\mathrm{e}}\left(\begin{array}{cc}
0 & 0 \\
0 & \vec{\sigma}
\end{array}\right) \cdot \vec{E}_{\mathrm{mol}}, \\
& W_{d} d_{\mathrm{e}}=2\left\langle{ }^{2} \Sigma_{1 / 2}\left|\sum_{i} \mathbf{H}_{d}(i)\right|^{2} \Sigma_{1 / 2}\right\rangle .
\end{aligned}
$$

The constant $\frac{1}{2} W_{d}$ characterizes an effective electric field on the unpaired electron. All the P-and P,T-odd constants $W_{X}$ depend on the electron spin density in the vicinity of the heavy nucleus. The reliability of their calculation can be tested by comparison of the calculated and experimental values for the hyperfine constants $A$ and $A_{d}$.

Details of calculations. Let us consider the scheme of calculation for the above molecules on example of the YbF calculation carried out mainly in paper 74]. The stage of the GRECP calculation of the valence structure for the ground state ${ }^{2} \Sigma$ of YbF was performed by analogy with the previous YbF calculation 72 . The main difference from the latter was the use of technique [46, 84] for freezing the $5 s$ and $5 p$ pseudospinors derived from the calculation on the $\mathrm{Yb}^{2+}$ cation. It was necessary to freeze these shells because their polarization was taken into account at the stage of calculating the effective operator (EO, see 79, 95] for details). The spin-averaged GRECP calculations in the framework of the RASSCF method 96, 97] with 5284 configurations were performed for 11 electrons distributed in RAS- $1=(2,0,0,0)$, RAS- $2=(2,1,1,0)$ and RAS-3 $=(6,4,4,2)$ active orbital subspaces according to the $A_{1}, B_{1}, B_{2}$ and $A_{2}$ irreducible representations (irreps) of the $C_{2 v}$ symmetry group used in the calculation (see paper [96] for details on the RASSCF method). Similar calculation was also carried out for the BaF molecule [3].

Expressions for the $W_{d}$ parameter which characterizes the internal molecular field are presented in Eqs. (15) and (16), whereas expressions for other electronic matrix elements which correspond to the parameters $A, A_{\mathrm{d}}$ and $W_{X}$ of operator (13) can be found in papers 65, 93. All the radial integrals and atomic four-component spinors were calculated for the finite nucleus ${ }^{171} \mathrm{Yb}$ using a model of a uniform charge distribution within a sphere. As is mentioned in the introduction, atomic matrix elements of operator (15) are proportional to $Z^{3}$. The same scaling is applicable to the constant $W_{\mathrm{S}}$, while the matrix elements which contribute to the constants $W_{\mathrm{A}}$ are proportional to $Z^{2}$. The nuclear charge of fluorine is eight times smaller than that of ytterbium, therefore, the contributions to the $W_{X}$ parameters from the vicinity of the fluorine nucleus can be neglected. The additional argument in favour of this approximation is connected with the circumstance that the state of the unpaired electron in the YbF molecule is described mainly by the ytterbium orbitals and thus the spin density is also localized near ytterbium.

Results. Results of the calculation for the spin-rotational Hamiltonian parameters are presented in Table $\$ Comparison of the results of the GRECP/RASSCF calculations 72] with the results of the GRECP/RASSCF/EO calculations 74 ] confirms the conclusion made in paper 72] that the core-valence correlations of the unpaired YbF electron 
occupying mainly the hybridized $6 s-6 p$ orbital of $\mathrm{Yb}$ with the $5 s$ and then with $5 p$ shells of the Yb core (first of all, spin polarization of the $5 s, 5 p$ shells) contribute very essentially to the hyperfine and the P-and P,T-odd constants.

Our final results for the hyperfine structure constant $A$ differ by less than $3 \%$ from the experimental value [98]. This means that the space-isotropic part of the unpaired spin density in the vicinity of the $\mathrm{Yb}$ nucleus in our calculations is obtained with rather good accuracy. However, it is also important to reproduce the anisotropic (dipole) part of the spin density which contributes to the dipole constant $A_{\mathrm{d}}$. The value for $A_{\mathrm{d}}$ obtained in the RASSCF/EO calculation is in slightly better agreement with the experimental datum than the value from paper 75 but is still underestimated by $23 \%$. About one half of this difference can be explained by the fact that the $4 f$ shell of $\mathrm{Yb}$ was frozen in our molecular calculation (it was first pointed out by Khriplovich that accounting for excitations from the $4 f$-shell can be important). In particular, these excitations can explain the small value of the spin-doubling constant $\gamma$ [99, 100]. It was shown in semiempirical calculation [95] that the contribution of the $4 f$-shell excitations to the spin density can result in a significant correction to the constant $A_{\mathrm{d}}$. Using Eqs. (19) and (31) from paper [95], one can go to the following estimates for the contributions from the $4 f$-shell excitations to $A$ and $A_{\mathrm{d}}$ :

$$
\delta A \approx-3 \mathrm{MHz}, \quad \delta A_{\mathrm{d}} \approx 15 \mathrm{MHz}
$$

Note that the semiempirical corrections obtained in [74] arise from the admixture to the molecular wavefunction of the configuration with the hole in the $4 f$-shell. The weight of this configuration was estimated in paper [95] to be of the order of $4 \%$. This admixture is a purely molecular effect and is not described by the effective operator technique. Thus, one can conclude that the $\delta A_{\mathrm{d}}$ value can be added to the RASSCF/EO value for $A_{\mathrm{d}}$, resulting in $A_{\mathrm{d}} \approx 94 \mathrm{MHz}$ which is essentially closer to the experimental value of $102 \mathrm{MHz}$. Note also that ab initio correlation calculation of $\mathrm{YbF}$ with, at least, $4 f$ shell treated explicitly should be performed to check the above described mechanism of increasing the calculated $A_{\mathrm{d}}$ value. It is likely that the real situation is more complicated and indirect contributions to $A_{\mathrm{d}}$ caused by the $4 f$ shell correlation-relaxation effects and by other higher order contributions (which are not accounted for in the considered calculation) are mainly responsible for the obtained underestimation of $A_{\mathrm{d}}$.

It is essential that similar contribution of the $4 f$-shell excitations to the constant $W_{d}$ is strongly suppressed. Indeed, operator (15) mixes $f$-and $d$-waves. The $4 d$-shell is localized rather deeply in the ytterbium core and its mixture with the $4 f$-orbitals by the molecular field is very small while the $5 d$ electrons are relatively weakly-bound and does not penetrate essentially into the region of the $4 f$-shell localization. Similar contributions to other constants $W_{X}$ are negligible due to the contact character of the corresponding interactions.

One can see that the values of the $W_{d}$ constant from the unrestricted DHF calculation (accounting for the spinpolarization of core shells) [6] , the latest semiempirical calculation [95] and the GRECP/RASSCF/NOCR/EO calculation are in very good agreement. It is also important for reliability of the carried out calculations that the contribution from the valence electron to $W_{d}$ in paper 76 ] differs only by $7.4 \%$ from the corresponding GRECP/SCF/NOCR calculation [72] (see Table I). Another DHF calculation 75] gives a value that is about two times smaller (that is rather explained by different definitions used for $W_{d}$ ).

Similar increase in the values for the hyperfine constants and parameters of the P,T-odd interactions when the correlations with the core shells (first of all, $5 s, 5 p$ ) are taken into account is also observed for the BaF molecule 73. as one can see in Table प). Of course, the corrections on the $4 f$-electron excitations are not required for this molecule. The enhancement factor for the P,T-odd effects in the BaF molecule is three times smaller than in $\mathrm{YbF}$ mainly because of smaller nuclear charge of $\mathrm{Ba}$. Therefore, barium fluoride can be considered as a less promising molecule for a search for the PNC effects. 
TABLE I: Parameters of the spin-rotational Hamiltonian for the ${ }^{171} \mathrm{YbF}$ and ${ }^{137} \mathrm{BaF}$ molecules; $A=\left(A_{\|}+2 A_{\perp}\right) / 3$ (isotropic) and $A_{d}=\left(A_{\|}-A_{\perp}\right) / 3$ (dipole) are the HFS constants.

\begin{tabular}{|c|c|c|c|c|c|}
\hline & $\begin{array}{c}A \\
(\mathrm{MHz})\end{array}$ & $\begin{array}{c}A_{d} \\
(\mathrm{MHz})\end{array}$ & $\begin{array}{c}W_{d} \\
\left(10^{25} \frac{\mathrm{Hz}}{\mathrm{ecm}}\right)\end{array}$ & $\begin{array}{c}W_{A} \\
(\mathrm{kHz})\end{array}$ & $\begin{array}{l}W_{S} \\
(\mathrm{~Hz})\end{array}$ \\
\hline \multicolumn{6}{|c|}{ The ${ }^{171} \mathbf{Y b F}$ molecule } \\
\hline Experiment [98] & 7617 & 102 & & & \\
\hline Semiempirical [101] & & & -1.5 & 730 & -48 \\
\hline Semiempirical [73] (with $4 f$-correction) & & & -1.26 & & -43 \\
\hline GRECP/SCF/NOCR [72] & 4932 & 59 & -0.91 & 484 & -33 \\
\hline GRECP/RASSCF/NOCR [72] & 4854 & 60 & -0.91 & 486 & -33 \\
\hline Restricted DHF (Quiney, 1998) [75] & 5918 & 35 & -0.31 & 163 & -11 \\
\hline Restricted DHF + core polarization & 7865 & 60 & -0.60 & 310 & -21 \\
\hline Rescaled restricted DHF & & & -0.62 & 326 & -22 \\
\hline \multicolumn{6}{|l|}{ Unrestricted DHF (Parpia, 1998) [76] } \\
\hline (unpaired valence electron) & & & -0.962 & & \\
\hline Unrestricted DHF [76] & & & -1.203 & & -22 \\
\hline GRECP/RASSCF/NOCR/EO [74] & 7842 & 79 & -1.206 & 634 & \\
\hline \multicolumn{6}{|l|}{$\begin{array}{l}\text { GRECP/RASSCF/NOCR/EO [74] } \\
\text { (with } 4 \text { f-correction) }\end{array}$} \\
\hline \multicolumn{6}{|c|}{ The ${ }^{137} \mathrm{BaF}$ molecule } \\
\hline Experiment $[102]^{a}$ & 2326 & 25 & & & \\
\hline Semiempirical [20] & & & -0.41 & 240 & -13 \\
\hline Experiment $[103]^{b}$ & 2418 & 17 & & & \\
\hline Semiempirical [20] & & & -0.35 & 210 & -11 \\
\hline GRECP/SCF/NOCR [73] & 1457 & 11 & -0.230 & 111 & -6.1 \\
\hline GRECP/RASSCF/NOCR [73] & 1466 & 11 & -0.224 & 107 & -5.9 \\
\hline GRECP/SCF/NOCR/EO [73] & 2212 & 26 & -0.375 & 181 & \\
\hline GRECP/RASSCF/NOCR/EO [73] & 2224 & 24 & -0.364 & 175 & \\
\hline
\end{tabular}

${ }^{a}$ The HFS constants are measured in an inert gas matrix 102$]$ and the semiempirical $W_{X}$ values 20$]$ are based on these data.

${ }^{b}$ The HFS constants were measured in a molecular beam [103].

\section{CALCULATION OF ${ }^{205}$ TLF MOLECULE.}

Effective Hamiltonian. Here we consider the interaction of the EDM of unpaired proton in ${ }^{205} \mathrm{Tl}$ with the internal electromagnetic field of the ${ }^{205} \mathrm{TlF}$ molecule [14]. This molecule is one of the best objects for the proton EDM measurements. The effective interaction with the proton EDM in TlF is written in the form

$$
H^{\mathrm{eff}}=\left(d^{V}+d^{M}\right) \vec{I} / I \cdot \vec{\lambda}
$$

where $\vec{I}$ is the Tl nuclear spin operator, $\vec{\lambda}$ is the unit vector along $z$ (from Tl to $\mathrm{F}$ ), $d^{V}$ and $d^{M}$ are volume and magnetic constants [104]

$$
d^{V}=6 S X=\left(-d_{p} R+Q\right) X
$$


$S$ is the nuclear Schiff moment, $d_{p}$ is the proton EDM,

$$
X=\frac{2 \pi}{3}\left[\frac{\partial}{\partial z} \rho_{\psi}(\vec{r})\right]_{x, y, z=0}
$$

$\rho_{\psi}(\vec{r})$ is the electronic density calculated from the electronic wave function $\psi$;

$$
d^{M}=2 \sqrt{2}\left(d_{p}+d_{N}\right)\left(\frac{\mu}{Z}+\frac{1}{2 m c}\right) M
$$

where $d_{N}$ is the nuclear EDM arising due to P,T-odd nuclear forces; $\mu, m$ and $Z$ are the magnetic moment, mass and charge of the $\mathrm{Tl}$ nucleus; $c$ is the velocity of light,

$$
M=\frac{1}{\sqrt{2}}\left\langle\psi\left|\sum_{i}\left(\frac{\vec{\alpha}_{i} \times \overrightarrow{\mathbf{l}}_{i}}{r_{i}^{3}}\right)_{z}\right| \psi\right\rangle,
$$

$\overrightarrow{\mathbf{l}_{\mathbf{i}}}$ is the orbital momentum for electron $i ; \vec{\alpha}_{i}$ are its Dirac matrices. $R$ and $Q$ are factors determined by the nuclear structure of ${ }^{205} \mathrm{Tl}$ :

$$
\begin{array}{r}
R=\left\langle\psi_{N}\left(\vec{r}_{n}\right)\left|\sum_{n}\left(q_{n} / Z-\delta_{n, 3 s}\right) r_{n}^{2}\right| \psi_{N}\left(\vec{r}_{n}\right)\right\rangle, \\
Q=\left[3 / 5\left\langle\psi_{N}\left(\vec{r}_{n}\right)\left|\sum_{n}\left(q_{n} \vec{r}_{n}\right)\right| \psi_{N}\left(\vec{r}_{n}\right)\right\rangle-\right. \\
1 / Z\left\langle\psi_{N}\left(\vec{r}_{n}\right)\left|\sum_{n}\left(q_{n} r_{n}^{2}\right)\right| \psi_{N}\left(\vec{r}_{n}\right)\right\rangle \\
\left.\times\left\langle\psi_{N}\left(\vec{r}_{n}\right)\left|\sum_{n}\left(q_{n} \vec{r}_{n}\right) / r_{n}^{2}\right| \psi_{N}\left(\vec{r}_{n}\right)\right\rangle\right]_{\vec{I}},
\end{array}
$$

where $\psi_{N}\left(\vec{r}_{n}\right)$ is the nuclear wave function.

Accounting for $H_{\text {eff }}$ leads to different hyperfine splitting of TIF in parallel and antiparallel electric and magnetic fields. The level shift $h \nu=4\left(d^{V}+d^{M}\right)\langle\vec{I} / I \cdot \vec{\lambda}\rangle$ is measured experimentally (for the latest results on TIF see 13$]$ ).

The parameters $X$ of Eq. (20) and $M$ of Eq. (22) are determined by the electronic structure of the molecule. They were calculated recently for the ground $0^{+}$state of TIF by Parpia [69] and by Laerdahl, Saue, Faegri Jr., and Quiney 68], using the Dirac-Fock method with gaussian basis sets of large sizes (see Table 1 ). (Below we refer to paper 105] with the calculations presented in details and not to the brief communication 68] of the same authors.) In paper [14] the first calculation of ${ }^{205} \mathrm{TlF}$ that accounts for correlation effects was performed (see also [106] where the limit on the Schiff moment of ${ }^{205} \mathrm{Tl}$ was recalculated).

Details of calculations. In paper 14] 21-electron GRECP 107] for Tl was used. The correlation spin-orbital basis set consisted of $26 s, 25 p, 18 d, 12 f$, and $10 g$ gaussian-type orbitals on Tl, contracted to $6 s 6 p 4 d 2 f 1 g$. The basis was optimized in a series of atomic two-component GRECP calculations, with correlation included by the relativistic coupled cluster method [108] with single and double cluster amplitudes; the average energy of the two lowest states of the atom was minimized. The basis set generation procedure is described in Refs. 109, 110. The basis set was designed to describe correlation in the outer core $5 s$ and $5 p$ shells of $\mathrm{Tl}$, in addition to the $5 d$ and valence shells. While correlating the $5 s$ and $5 p$ shells may not be important for a majority of chemical and physical properties of the atom, it is essential for describing properties coming from the inner Tl region, including P,T-odd effects. The $(14 s 9 p 4 d 3 f) /[4 s 3 p 2 d 1 f]$ basis set from the ANO-L library 97$]$ is used for fluorine.

First, one-component SCF calculation of the $(1 \sigma \ldots 7 \sigma)^{14}(1 \pi 2 \pi 3 \pi)^{12}(1 \delta)^{4}$ ground state of TlF is performed, using the spin-averaged GRECP for $\mathrm{Tl}$ which simulates the interactions of the valence and outer core $(5 s 5 p 5 d)$ electrons with the inner core $[\mathrm{Kr}] 4 d_{3 / 2}^{4} 4 d_{5 / 2}^{6} 4 f_{5 / 2}^{6} 4 f_{7 / 2}^{8}$. This is followed by two-component RCC calculations, with only single 
(RCC-S) or with single and double (RCC-SD) cluster amplitudes. The RCC-S calculations with the spin-dependent GRECP operator take into account effects of spin-orbit interaction at the level of the one-configurational SCF-type method. The RCC-SD calculations include, in addition, the most important electron correlation effects.

At the restoration stage, the nucleus was modeled as a uniform charge distribution within a sphere with radius $r_{\text {nucl }}=7.1 \mathrm{fm} \equiv 1.34 \times 10^{-4}$ a.u., whereas previous calculations $[69$, 105] employed a spherical gaussian nuclear charge distribution (the root mean square radius in all calculations is $5.5 \mathrm{fm}$, in accord with the parametrization of Johnson and Soff [11] , and agrees with the experimental value $5.483 \mathrm{fm}$ for the ${ }^{205} \mathrm{Tl}$ nucleus [112]). The two equivalent $[15 s 12 p 12 d 8 f]$ numerical basis sets were generated for the restoration.

The quality of the approximation for the two-center molecular spinors and, consequently, of the calculated properties increases with the value of $L_{\max }$. A series of calculations of the $M$ parameter was performed using Eq. (7) with basis functions including up to $p, d$ and $f$ harmonics. It is found (see Table III) that accounting for only $s$ and $p$ functions in the expansion determines $M$ with $90 \%$ accuracy. Because the contribution of $f$ functions is only about $0.3 \%$ and amplitudes of higher harmonics on the nucleus are suppressed by the leading term $\sim r^{(j-1 / 2)}$, the error due to the neglect of spherical harmonics beyond $f$ is estimated to be below $0.1 \%$. Calculation of the $X$ parameter requires $s$ and $p$ harmonics (see Ref. 105]), although, strictly speaking, $d$ harmonics also give nonzero contributions.

In the RCC calculations, the $X$ and $M$ parameters were calculated by the finite field method (e.g., see Refs. 91, 92]). The operator corresponding to a desired property (Eqs. (20) and (22)) is multiplied by a small parameter $\lambda$ and added to the Hamiltonian. The derivative of the energy with respect to $\lambda$ gives the computed property. This is strictly correct only at the limit of vanishing $\lambda$, but it is usually possible to find a range of $\lambda$ values where the energy is almost linear with $\lambda$ and energy changes are large enough to allow sufficient precision. The quadratic dependence on $\lambda$ is eliminated in the present calculations by averaging absolute energy changes obtained with $\lambda$ of opposite signs.

Results. Calculations were carried out at two internuclear separations, the equilibrium $R_{e}=2.0844 \AA$ as in Ref. 69], and $2.1 \AA$, for comparison with Ref. [105]. The results are collected in Table [I] The first point to notice is the difference between spin-averaged SCF values and RCC-S values, the latter include spin-orbit interaction effects. These effects increase $X$ by $9 \%$ and decrease $M$ by $21 \%$. The RCC-S function can be written as a single determinant, and results may therefore be compared with DHF values, even though the RCC-S function is not variational. GRECP/RCC-S/NOCR values of the $M$ parameter are indeed within $3 \%$ and $1 \%$ of difference with the corresponding DHF values [69, 105] (Table III). This agreement confirms the validity of the used approximations. In particular, freezing the inner core shells is justified, as inner core relaxation effects have little influence on the properties calculated here, a conclusion already drawn by Quiney et al. 105].

Much larger differences occur for the $X$ parameter. There are also large differences between the two DHF calculations for $X$, which cannot be explained by the small change in internuclear separation. The value of $X$ may be expected to be less stable than $M$, because it is determined by the derivative of the electronic density at the $\mathrm{Tl}$ nucleus and involves large cancellations 105] between contributions of large and small components, each of them is about 20 times larger than their sum. Thus, a strong dependence of $X$ on the basis used may be expected. The DHF values collected in Table [1 indeed show such dependence. Results obtained in Refs. 69] and [105] with comparable even-tempered basis sets, $(28 s 28 p 12 d 8 f)$ and $(28 s 28 p 14 d 8 f)$, are rather close, differing by 340 a.u. Improving the Tl basis to $(34 s 34 p 16 d 9 f)$ 105] increases $X$ by 650 a.u. or $8 \%$. Further improvement of the basis may be expected to yield even higher $X$ values. The numerical basis functions obtained in atomic DHF calculations and used for the restoration are highly accurate near the nucleus, so that the GRECP/RCC-S/NOCR value for $X$, which is higher than that of Quiney et al. 105, seems reasonable. The different nuclear models used in the present and DHF [69, 105] calculations may also contribute to the disagreement in $X$, which is determined by the derivative of the electronic charge density at a single point, the $\mathrm{Tl}$ origin. $M$ is affected by $\psi$ in a broader region, and is therefore far less sensitive 
TABLE II: Calculated $X$ (20) and $M$ (22) parameters (in a.u.) for the ${ }^{205} \mathrm{TlF}$ ground state, compared with DHF values 69 , 105]. GRECP/RCC-S results include spin-orbit interaction, and GRECP/RCC-SD values also account for electron correlation.

\begin{tabular}{|c|c|c|c|c|c|c|c|c|}
\hline & & \multicolumn{4}{|c|}{$R_{e}=2.0844 \AA$} & \multicolumn{3}{|c|}{$R=2.1 \AA$} \\
\hline \multicolumn{2}{|c|}{ Expansion } & \multicolumn{3}{|c|}{$M$} & $\begin{array}{r}s, p \\
X\end{array}$ & $\mathrm{~s}, \mathrm{p}$ & & $\begin{array}{r}s, p \\
X\end{array}$ \\
\hline \multicolumn{2}{|c|}{$\mathrm{SCF}$ (spin-averaged) } & 19.67 & 17.56 & 17.51 & 8967 & 19.52 & 17.43 & 8897 \\
\hline \multicolumn{2}{|c|}{ GRECP/RCC-S } & 16.12 & & 13.84 & 9813 & 16.02 & 13.82 & 9726 \\
\hline DHF [69] & Tl: $(28 s 28 p 12 d 8 f)$ & 15.61 & & & 7743 & & & \\
\hline \multirow[t]{4}{*}{ DHF [105] } & $\mathrm{Tl}:(25 s 25 p 12 d 8 f)$ & & & & & & $13.64^{a}$ & 8098 \\
\hline & Tl: $(28 s 28 p 14 d 8 f)$ & & & & & & $13.62^{a}$ & 8089 \\
\hline & $\mathrm{Tl}:(31 s 31 p 15 d 8 f)$ & & & & & & $13.66^{a}$ & 8492 \\
\hline & Tl:(34s34p16d9f) & & & & & & $13.63^{a}$ & 8747 \\
\hline \multicolumn{2}{|c|}{ GRECP/RCC-SD } & & & 11.50 & 7635 & & & \\
\hline
\end{tabular}

${ }^{a} M$ is calculated in Ref. 105] using two-center molecular spinors, corresponding to infinite $L_{\max }$ in Eq. (7).

to the nuclear model.

The electron correlation effects are calculated by the RCC-SD method at the molecular equilibrium internuclear distance $R_{e}$. A major correlation contribution is observed, decreasing $M$ by $17 \%$ and $X$ by $22 \%$.

The hyperfine structure constants of $\mathrm{Tl} 6 p_{1 / 2}^{1}$ and $\mathrm{Tl}^{2+} 6 s^{1}$, which (like $X$ and $M$ ) depend on operators concentrated near the $\mathrm{Tl}$ nucleus, were also calculated. The errors in the DF values are 10-15\%; the RCC-SD results are within 1-4\% of experiment. The improvement in $X$ and $M$ upon inclusion of correlation is expected to be similar.

\section{CALCULATIONS OF ${ }^{207}$ PBO MOLECULE.}

It was noted previously that the experiments on the excited $a(1)$ 23] or $B(1)$ [113] states of $\mathrm{PbO}$ having nonzero projection of total electronic momentum on the internuclear axis can be, in principle, sensitive enough to detect $d_{e}$ three or even four orders of magnitude lower than the current limit. The knowledge of the effective electric field, $W_{d}$, seen by an unpaired electron is required for extracting $d_{e}$ from the measurements. In papers $77,78, W_{d}$ for the $a(1)$ and $B(1)$ states of the $\mathrm{PbO}$ molecule was calculated by the RCC-SD [80, 81] and CI [114, 82, 83, 84] methods. To provide an accuracy check in calculation of the electronic structure near the $\mathrm{Pb}$ nucleus the hyperfine constant, $A_{\|}$, was also calculated.

Details of calculations. The 22-electron GRECP for $\mathrm{Pb}$ [107] is used at the first stage of the two-step calculations of $\mathrm{PbO}$ : the inner shells of the $\mathrm{Pb}$ atom $(1 s$ to $4 f)$ are absorbed into the GRECP, and the $5 s 5 p 5 d 6 s 6 p$ electrons and all the oxygen electrons are treated explicitly at the integral preparation part. Two series of RCC-SD calculations with 10 (when freezing the $5 s 5 p 5 d$ shells of lead employing the level shift technique [46] and freezing the $1 s$ shell of oxigen after the RASSCF stage) and all 30 electrons treated explicitly were performed. Only 10 electrons were treated explicitly in the CI calculations. For each series of calculations, correlation spin-orbital basis sets are optimized in atomic two-component GRECP/RCC calculations of $\mathrm{Pb}$. The generated basis sets on $\mathrm{Pb}$ are $(14 s 18 p 16 d 8 f) /[4 s 7 p 5 d 3 f]$ for 30 -electron and $(15 s 16 p 12 d 9 f) /[5 s 7 p 4 d 2 f]$ for 10-electron calculations. The correlationconsistent $(10 s 5 p 2 d 1 f) /[4 s 3 p 2 d 1 f]$ basis listed in the MOLCAS 4.1 library [97] is used for oxygen. We found that the $f$ basis function of oxygen has little effect on the core properties calculated here and it was not included into the RCC calculations to reduce expenses.

The leading $\Lambda \Sigma$ coupling terms and configurations for the $a(1)$ and $B(1)$ states are ${ }^{3} \Sigma^{+} \sigma_{1}^{2} \sigma_{2}^{2} \sigma_{3}^{2} \pi_{1}^{3} \pi_{2}^{1}$ and ${ }^{3} \Pi_{1}$ $\sigma_{1}^{2} \sigma_{2}^{2} \sigma_{3}^{1} \pi_{1}^{4} \pi_{2}^{1}$, correspondingly. The molecular orbitals used in the CI and RCC-SD calculations are obtained by the 
RASSCF method [96, 97] with the spin-averaged GRECP part [46], i.e. only scalar-relativistic interactions are taken into account in the RASSCF calculation. The $a(1)$ state is of practical interest first of all and, therefore, the lowest ${ }^{3} \Sigma^{+}$state was calculated at this stage. Using the $C_{2 v}$ point group classification scheme, two $\mathrm{A}_{1}$ orbitals are in RAS1, six orbitals (two $\mathrm{A}_{1}$, two $\mathrm{B}_{1}$, and two $\left.\mathrm{B}_{2}\right)$ in RAS2, and $50\left(20 \mathrm{~A}_{1}, 6 \mathrm{~A}_{2}, 12 \mathrm{~B}_{1}\right.$, and $\left.12 \mathrm{~B}_{2}\right)$ in RAS3 for 10-electron calculation. $2 \mathrm{~A}_{1}$ orbitals are in RAS1, 6 orbitals $\left(2 \mathrm{~A}_{1}, 2 \mathrm{~B}_{1}\right.$, and $\left.2 \mathrm{~B}_{2}\right)$ in RAS2, and $41\left(16 \mathrm{~A}_{1}, 5 \mathrm{~A}_{2}, 10 \mathrm{~B}_{1}\right.$, and $10 \mathrm{~B}_{2}$ ) in RAS3 for 30-electron calculation. No more than two holes in RAS1 and two particles in RAS3 are allowed.

At the restoration stage the nucleus is modeled by a uniform charge distribution within a sphere of radius $r_{\text {nucl }}=$ $7.12 \mathrm{fm}=1.35 \times 10^{-4}$ a.u. The root mean square radius of the nucleus is $5.52 \mathrm{fm}$, in accord with the parametrization of Johnson and Soff 111], and agrees with the experimental value of $5.497 \mathrm{fm}$ 112] for the ${ }^{207} \mathrm{~Pb}$ nucleus. Taking this value for the root mean square radius and a Fermi distribution for the nuclear charge changes $A_{\|}$and $W_{d}$ by $0.1 \%$ or less. The equivalent basis sets generated are $[9 s 14 p 7 d]$ for 10 and $[6 s 7 p 5 d]$ for 30-electron calculation correspondingly.

Then the RCC-SD method and the spin-orbit CI approach with the selected single- and double-excitations from some multiconfigurational reference states ("mains") [84, 114] are employed in the sets of different $\Lambda$ S many-electron spin- and space-symmetry adapted basis functions

Results. CI calculations [78] were performed at two internuclear distances, $R=3.8$ a.u., as is in RCC calculations, and $R=4.0$ a.u. (in the RCC calculations [77] the only internuclear distance $R=3.8$ a.u. was used because of problem with convergence at larger distances). The calculated values with the one-center expansion of the molecular spinors in the $\mathrm{Pb}$ core on either $s, s ; p$ or $s ; p ; d$ partial waves are collected in Table III

Let us consider the $5 s, 5 p, 5 d$ orbitals of lead and $1 s$ orbital of oxigen as the outercore ones and $\sigma_{1}, \sigma_{2}, \sigma_{3}, \pi_{1}$, $\pi_{2}$ orbitals of the $\mathrm{PbO}$ (consisting mainly of $6 s, 6 p$ orbitals of $\mathrm{Pb}$ and $2 s, 2 p$ orbitals of $\mathrm{O}$ ) as valence. Although in the CI calculations we take into account only the correlation between valence electrons, the accuracy attained in the CI calculation of $A_{\|}$is much better than in the RCC-SD calculation. The main problem with the RCC calculation was that the used Fock-space RCC-SD version was not optimal in accounting for the nondynamic correlations (see 109] for details of RCC-SD and CI calculations of the Pb atom), though the potential of the RCC approach for electronic structure calculations is very high in prospect, especially, in the framework of the intermediate Hamiltonian formulation [81].

Then we estimate the contribution from correlations of valence electrons with outercore ones (which also account for correlations between outercore pairs of electrons) as difference in the results of the corresponding 10- and 30electron GRECP/RCC calculations (see also [109] where this correction is applied to the $\mathrm{Pb}$ atom). We designate such correlations in Table [II as "outercore correlations". When taking into account outercore contributions at the point $R=4.0$ a.u. we used the results of the RCC calculation at the point $R=3.8$ a.u. Since these contributions are relatively small and because the correlations with the outercore electrons are more stable than correlations in the valence region when the internuclear distance is changed the used approximation seems us reasonable. At last, the linear extrapolation of the results of calculations to the experimental equilibrium distances, $R_{e}=4.06$ a.u. for $a(1)$ 115] and $R_{e}=3.91$ a.u. for $B(1)[116]$ is applied. The final results are: $A_{\|}=-3826 \mathrm{MHz}, W_{d}=-6.1 \cdot 10^{24} \mathrm{~Hz} /(e \cdot \mathrm{cm})$ for $a(1)$ and $A_{\|}=4887 \mathrm{MHz}, W_{d}=-8.0 \cdot 10^{24} \mathrm{~Hz} /(e \cdot \mathrm{cm})$ for $B(1)$ states. The estimated error for the final $W_{d}$ parameter is $20 \%$ for $B(1)$ state. For $a(1)$ the estimated error bounds put the actual $W_{d}$ value between $90 \%$ and $130 \%$ of the final value (for details see [78]). 
TABLE III: Calculated parameters $A_{\|}$(in $\left.\mathrm{MHz}\right)$ and $W_{d}$ (in $10^{24} \mathrm{~Hz} /(e \cdot \mathrm{cm})$ ) for the $a(1)$ and $B(1)$ states of ${ }^{207} \mathrm{PbO}$ in the internuclear distances 3.8 and 4.0 a.u. The experimental value of $A_{\|}$in $a(1)$ is $-4113 \mathrm{MHz}$ [17]. The preliminary experimental value of $A_{\|}$for $B(1)$ is $5000 \pm 200 \mathrm{MHz}[118]$.

\begin{tabular}{|c|c|c|c|c|c|c|c|c|c|c|}
\hline \multirow{3}{*}{$\begin{array}{l}\text { State } \\
\text { Parameters } \\
\text { Expansion }\end{array}$} & \multirow{2}{*}{\multicolumn{3}{|c|}{$a(1) \quad \sigma_{1}^{2} \sigma_{2}^{2} \sigma_{3}^{2} \pi_{1}^{3} \pi_{2}^{1}$}} & \multirow{2}{*}{\multicolumn{2}{|c|}{${ }^{3} \Sigma_{1}$}} & \multicolumn{5}{|c|}{$B(1) \quad \sigma_{1}^{2} \sigma_{2}^{2} \sigma_{3}^{1} \pi_{1}^{4} \pi_{2}^{1} \quad{ }^{3} \Pi_{1}$} \\
\hline & \multicolumn{2}{|c|}{$A_{\|}$} & & & & \multicolumn{3}{|c|}{$A_{\|}$} & \multicolumn{2}{|c|}{$W_{d}$} \\
\hline & $\mathrm{s}$ & $\mathrm{s}, \mathrm{p}$ & $\mathrm{s}, \mathrm{p}, \mathrm{d}$ & $\mathrm{s}, \mathrm{p}$ & $\mathrm{s}, \mathrm{p}, \mathrm{d}$ & $\mathrm{s}$ & $\mathrm{s}, \mathrm{p}$ & $\mathrm{s}, \mathrm{p}, \mathrm{d}$ & $\mathrm{s}, \mathrm{p}$ & $\mathrm{s}, \mathrm{p}, \mathrm{d}$ \\
\hline \multicolumn{11}{|c|}{ Internuclear distance $R=3.8$ a.u. } \\
\hline 10e-RASSCF & -894 & -1505 & -1503 & 0.73 & 0.70 & & & & 0.0 & 0.0 \\
\hline 30e-RASSCF & -759 & -1705 & -1699 & 0.96 & 0.91 & & & 1900 & 0.0 & 0.0 \\
\hline 10e-RCC-SD [77] & & & -2635 & -2.93 & -3.05 & & & 3878 & -11.10 & -10.10 \\
\hline 30e-RCC-SD [77] & & & -2698 & & -4.10 & & & 4081 & -9.10 & -9.70 \\
\hline outercore (30e-RCC-SD - 10e-RCC-SD) & & & -63 & & -1.05 & & & 203 & & 0.40 \\
\hline 10e-CI [78] & & & -3446 & & -4.13 & & & 4582 & & -10.64 \\
\hline \multicolumn{11}{|l|}{ FINAL [78] } \\
\hline$(10 \mathrm{e}-\mathrm{CI}+$ outercore $)$ & & & -3509 & & -5.18 & & & 4785 & & -10.24 \\
\hline \multicolumn{11}{|c|}{ Internuclear distance $R=4.0$ a.u. } \\
\hline 10e-RASSCF & -770 & -1384 & -1383 & 1.05 & 1.00 & & & & 0.0 & 0.0 \\
\hline 10e-CI [78] & & & -3689 & & -4.81 & & & 4762 & & -7.18 \\
\hline \multicolumn{11}{|l|}{ FINAL [78] } \\
\hline$(10 \mathrm{e}-\mathrm{CI}+\text { outercore })^{a}$ & & & -3752 & & -5.86 & & & 4965 & & -6.78 \\
\hline
\end{tabular}

${ }^{a}$ It is assumed that the outercore contribution at the internuclear distance $R=4.0$ a.u. is approximately the same as is at $R=3.8$ a.u.

\section{CONCLUSIONS}

The P,T-parity nonconservation parameters and hyperfine constants are calculated for the heavy-atom molecules which are of primary interest for modern experiments on search for the PNC effects. It is found that accounting for the electron correlations is necessary for precise calculation of these properties. The developed two-step (GRECP/NOCR) scheme of calculation of the properties heavily concentrated in atomic cores is a very efficient way to take account of these correlations with moderate efforts. The results of the two-step calculations for the hyperfine constants differ less than $10 \%$ from the corresponding experimental data. The comparable level of accuracy is expected for the P,T-odd spin-rotational Hamiltonian parameters which can not be obtained experimentally. The precision of the discussed calculations is limited by the current possibilities of the correlation methods and codes and not by the GRECP and NOCR approximations, despite the GRECP/NOCR method allows one to reduce seriously the expenses of the correlation treatment as compared to conventional Dirac-Coulomb(-Breit) approaches when using basis of molecular spin-orbitals instead of spinors etc. In turn, the attained accuracy is sufficient for a reliable interpretation of the results measured in the molecular PNC experiments.

Acknowledgments. The present work is supported by the U.S. CRDF grant RP2-2339-GA-02 and RFBR grant 03-03-32335. A.P. is grateful to Ministry of education of Russian Federation (grant PD 02-1.3-236) and to St.Petersburg Committee of Science (grant PD 03-1.3-60). N.M. is also supported by the grants of Russian Science Support Foundation and the governor of Leningrad district.

[1] J. H. Christenson, J. W. Cronin, V. L. Fitch, and R. Turlay, Phys. Rev. Lett. 13, 138 (1964). 
[2] E. D. Commins, Adv. At. Mol. Opt. Phys. 40, 1 (1999).

[3] J. Sapirstein, in Relativistic Electronic Structure Theory. Part I. Fundamentals, edited by P. Schwerdtfeger (Elsevier, Amsterdam, 2002), pp. 468-522.

[4] R. Berger, in Relativistic Electronic Structure Theory. Part II. Applications, edited by P. Schwerdtfeger (Elsevier, Amsterdam, 2004), pp. 188-288, theoretical and Computational Chemistry, Vol. 14.

[5] J. S. M. Ginges and V. V. Flambaum, Phys. Rep. 397, 63 (2004).

[6] L. D. Landau, Sov. Phys.-JETP 5, 336 (1957).

[7] B. C. Regan, E. D. Commins, C. J. Schmidt, and D. DeMille, Phys. Rev. Lett. 88, 071805/1 (2002).

[8] M. V. Romalis and W. C. G. E. N. Fortson, Phys. Rev. Lett. 86, 2505 (2001).

[9] V. F. Dmitriev and R. A. Sen'kov, Phys. Rev. Lett. 91, 212303 (2003).

[10] P. G. H. Sandars, Phys. Rev. Lett. 19, 1396 (1967).

[11] P. G. H. Sandars, Physics Letters 14, 194 (1965).

[12] E. A. Hinds, C. E. Loving, and P. G. H. Sandars, Phys. Lett. B 62, 97 (1976).

[13] D. Cho, K. Sangster, and E. A. Hinds, Phys. Rev. A 44, 2783 (1991).

[14] A. N. Petrov, N. S. Mosyagin, T. A. Isaev, A. V. Titov, V. F. Ezhov, E. Eliav, and U. Kaldor, Phys. Rev. Lett. 88, 073001/1 (2002).

[15] L. N. Labzowsky, Sov. Phys.-JETP 48, 434 (1978).

[16] V. G. Gorshkov, L. N. Labzowsky, and A. N. Moskalev, Sov. Phys.-JETP 49, 414 (1979).

[17] O. P. Sushkov and V. V. Flambaum, Sov. Phys.-JETP 48, 608 (1978).

[18] O. P. Sushkov, V. V. Flambaum, and I. B. Khriplovich, Sov. Phys.-JETP 87, 1521 (1984).

[19] V. V. Flambaum and I. B. Khriplovich, Phys. Lett. A 110, 121 (1985).

[20] M. G. Kozlov, Sov. Phys.-JETP 62, 1114 (1985).

[21] A. V. Titov, Ph.D. thesis, St.-Petersburg (Leningrad) State University, Russia (1985).

[22] J. J. Hudson, B. E. Sauer, M. R. Tarbutt, and E. A. Hinds, Phys. Rev. Lett. 89, 023003/1 (2002).

[23] D. DeMille, F. Bay, S. Bickman, D. Kawall, D. Krause, Jr., S. E. Maxwell, and L. R. Hunter, Phys. Rev. A 61, 052507/1 (2000).

[24] I. P. Grant and H. M. Quiney, in Relativistic Electronic Structure Theory. Part I. Fundamentals, edited by P. Schwerdtfeger (Elsevier, Amsterdam, 2002), pp. 107-202.

[25] L. Visscher, in Relativistic Electronic Structure Theory. Part I. Fundamentals, edited by P. Schwerdtfeger (Elsevier, Amsterdam, 2002), pp. 291-331.

[26] W. Kutzelnigg, in Relativistic Electronic Structure Theory. Part I. Fundamentals, edited by P. Schwerdtfeger (Elsevier, Amsterdam, 2002), pp. 664-757.

[27] L. Visscher, Chem. Phys. Lett. 253, 20 (1996).

[28] L. Visscher, J. Comput. Chem. 23, 759 (2002).

[29] J. H. Wood and A. M. Boring, Phys. Rev. B 18, 2701 (1978).

[30] J. C. Barthelat, M. Pelissier, and P. Durand, Phys. Rev. A 21, 1773 (1980).

[31] E. van Lenthe, E. J. Baerends, and J. G. Snijders, J. Chem. Phys. 99, 4597 (1993).

[32] A. Wolf, M. Reiher, and B. A. Hess, in Relativistic Electronic Structure Theory. Part I. Fundamentals, edited by P. Schwerdtfeger (Elsevier, Amsterdam, 2002), pp. 622-663.

[33] Y. S. Lee, W. C. Ermler, and K. S. Pitzer, J. Chem. Phys. 67, 5861 (1977).

[34] W. C. Ermler, R. B. Ross, and P. A. Christiansen, Adv. Quantum Chem. 19, 139 (1988).

[35] P. Schwerdtfeger, in Theoretical chemistry and physics of heavy and superheavy elements, edited by U. Kaldor and S. Wilson (Kluwer academic publishers, Dordrecht, The Netherlands, 2003), vol. 11 of Progr. Theor. Chem. Phys., pp. $399-438$.

[36] G. Theurich and N. A. Hill, Phys. Rev. B 64, 073106, 1 (2001).

[37] W. Kutzelnigg, Int. J. Quantum Chem. 25, 107 (1984).

[38] L. Seijo, J. Chem. Phys. 102, 8078 (1995).

[39] P. E. Blöchl, Phys. Rev. B 41, 5414 (1990). 
[40] D. Vanderbilt, Phys. Rev. B 41, 7892 (1990).

[41] G. Kresse and D. Joubert, Phys. Rev. B 59, 1758 (1999).

[42] C. J. Pickard, B. Winkler, R. K. Chen, M. C. Payne, M. H. Lee, J. S. Lin, J. A. White, V. Milman, and D. Vanderbilt, Phys. Rev. Lett. 85, 5122 (2000).

[43] P. Durand and J.-C. Barthelat, Theor. Chim. Acta 38, 283 (1975).

[44] P. A. Christiansen, Y. S. Lee, and K. S. Pitzer, J. Chem. Phys. 71, 4445 (1979).

[45] D. R. Hamann, M. Schlüter, and C. Chiang, Phys. Rev. Lett. 43, 1494 (1979).

[46] A. V. Titov and N. S. Mosyagin, Int. J. Quantum Chem. 71, 359 (1999).

[47] A. V. Titov (2002), Doctorate Thesis, (Petersburg Nuclear Physics Institute, RAS, Russia) [in Russian].

[48] M. Dolg, in Modern Methods and Algorithms of Quantum Chemistry, edited by J. Grotendorst (Jülich, 2000), vol. 1, pp. 479-508, [http://www.fz-juelich.de/nic-series].

[49] A. V. Titov and N. S. Mosyagin, eprint http://xxx.lanl.gov/abs/physics/0008160, 2000, 18 p.

[50] A. V. Titov and N. S. Mosyagin, Russ. J. Phys. Chem. 74, Suppl.2, S376 (2000), [Eprint http://xxx.lanl.gov/ abs/physics/0008160].

[51] A. N. Petrov, N. S. Mosyagin, A. V. Titov, and I. I. Tupitsyn (2003), eprint http:// xxx.lanl.gov/abs/physics/0311026.

[52] N. S. Mosyagin and A. V. Titov (2004), eprint http:// xxx.lanl.gov/abs/physics/0406143.

[53] V. Bonifacic and S. Huzinaga, J. Chem. Phys. 60, 2779 (1974).

[54] S. Katsuki and S. Huzinaga, Chem. Phys. Lett. 152, 203 (1988).

[55] J. C. Phillips and L. Kleinman, Phys. Rev. 116, 287 (1959).

[56] L. F. Pacios and P. A. Christiansen, J. Chem. Phys. 82, 2664 (1985).

[57] P. E. Blöchl, Phys. Rev. B 50, 17953 (1994).

[58] A. V. Titov, in Theses of reports of the 4th European Conf. on Atomic and Mol. Physics (Riga, Latvia, 1992$)$, p. 299.

[59] A. V. Titov, Int. J. Quantum Chem. 57, 453 (1996).

[60] J. P. Desclaux and P. Pyykkö, Chem. Phys. Lett. 29, 534 (1974).

[61] J. P. Desclaux and P. Pyykkö, Chem. Phys. Lett. 39, 300 (1976).

[62] J.-P. Desclaux, in Relativistic Electronic Structure Theory. Part I. Fundamentals, edited by P. Schwerdtfeger (Elsevier, Amsterdam, 2002), pp. 1-22.

[63] K. S. Pitzer, Acc. Chem. Res. 12, 271 (1979).

[64] P. Pyykkö and J.-P. Desclaux, Acc. Chem. Res. 12, 276 (1979).

[65] M. G. Kozlov, V. I. Fomichev, Y. Y. Dmitriev, L. N. Labzovsky, and A. V. Titov, J. Phys. B 20, 4939 (1987).

[66] E. A. Hinds and P. G. H. Sandars, Phys. Rev. A 21, 471 (1980).

[67] P. V. Coveney and P. G. H. Sandars, J. Phys. B 16, 3727 (1983).

[68] J. K. Laerdahl, T. Saue, K. Faegri, Jr, and H. M. Quiney, Phys. Rev. Lett. 79, 1642 (1997).

[69] F. A. Parpia, J. Phys. B 30, 3983 (1997).

[70] Y. Y. Dmitriev, Y. G. Khait, M. G. Kozlov, L. N. Labzovsky, A. O. Mitrushenkov, A. V. Shtoff, and A. V. Titov, Phys. Lett. A 167, 280 (1992).

[71] A. V. Titov, Int. J. Quantum Chem. 42, 1711 (1992).

[72] A. V. Titov, N. S. Mosyagin, and V. F. Ezhov, Phys. Rev. Lett. 77, 5346 (1996).

[73] M. G. Kozlov, A. V. Titov, N. S. Mosyagin, and P. V. Souchko, Phys. Rev. A 56, R3326 (1997).

[74] N. S. Mosyagin, M. G. Kozlov, and A. V. Titov, J. Phys. B 31, L763 (1998).

[75] H. M. Quiney, H. Skaane, and I. P. Grant, J. Phys. B 31, L85 (1998).

[76] F. Parpia, J. Phys. B 31, 1409 (1998).

[77] T. A. Isaev, A. N. Petrov, N. S. Mosyagin, A. V. Titov, E. Eliav, and U. Kaldor, Phys. Rev. A 69, 030501(R)/1 (2004).

[78] A. N. Petrov, A. V. Titov, T. A. Isaev, N. S. Mosyagin, and D. P. DeMille (2004), eprint http://xxx.lanl.gov/ abs/physics/0409045.

[79] V. A. Dzuba, V. V. Flambaum, and M. G. Kozlov, JETP Lett. 63, 882 (1996).

[80] U. Kaldor, in Recent Advances in Coupled-Cluster Methods, edited by R. J. Bartlett (World Scientific, Singapore, 1997), 
pp. $125-153$.

[81] A. Landau, E. Eliav, and U. Kaldor, Adv. Quantum Chem. 39, 171 (2001).

[82] R. J. Buenker and S. Krebs, in Recent Advances in Multireference Methods, edited by K. Hirao (World Scientific, Singapore, 1999), pp. 1-29.

[83] A. B. Alekseyev, H.-P. Liebermann, and R. J. Buenker, in Recent Advances in Relativistic Molecular Theory, edited by K. Hirao and Y. Ishikawa (World Scientific, Singapore, 2004), vol. 5 of Recent Advances in Comput. Chemistry.

[84] A. V. Titov, N. S. Mosyagin, A. B. Alekseyev, and R. J. Buenker, Int. J. Quantum Chem. 81, 409 (2001).

[85] V. F. Bratzev, G. V. Deyneka, and I. I. Tupitsyn, Bull. Acad. Sci. USSR, Phys. Ser. 41, 2655 (1977).

[86] I. I. Tupitsyn and A. N. Petrov, in 5-th Session of the V.A. Fock School on Quantum and Computational Chemistry (Novgorod the Great, 2002), p. 62.

[87] I. I. Tupitsyn (2003), program for atomic finite-difference four-component Dirac-Hartree-Fock-Breit calculations written on the base of the HFD code [85].

[88] I. I. Tupitsyn and N. S. Mosyagin (1995), program for atomic finite-difference two-component Hartree-Fock calculations with the generalized RECP in the $j j$-coupling scheme.

[89] I. I. Tupitsyn, N. S. Mosyagin, and A. V. Titov, J. Chem. Phys. 103, 6548 (1995).

[90] I. Lindgren, Rep. Prog. Phys. 47, 345 (1984).

[91] D. Kunik and U. Kaldor, J. Chem. Phys. 55, 4127 (1971).

[92] H. J. Monkhorst, Int. J. Quantum Chem.: Quantum Chem. Symp. 11, 421 (1977).

[93] M. Kozlov and L. Labzowsky, J. Phys. B 28, 1931 (1995).

[94] I. B. Khriplovich, Parity non-conservation in atomic phenomena (Gordon and Breach, New York, 1991).

[95] M. G. Kozlov and S. G. Porsev, Sov. Phys.-JETP 84, 461 (1997).

[96] J. Olsen and B. O. Roos, J. Chem. Phys. 89, 2185 (1988).

[97] K. Andersson, M. R. A. Blomberg, M. P. Fülscher, G. Karlström, R. Lindh, P.-A. Malmqvist, P. Neogrády, J. Olsen, B. O. Roos, A. J. Sadlej, et al. (1999), quantum-chemical program package "MOLCAS", Version 4.1.

[98] L. B. Knight, Jr. and W. Weltner, Jr., J. Chem. Phys. 53, L4111 (1970).

[99] B. E. Sauer, J. Wang, and E. A. Hinds, Phys. Rev. Lett. 74, 1554 (1995).

[100] B. E. Sauer, J. Wang, and E. A. Hinds, J. Chem. Phys. 105, 7412 (1996).

[101] M. G. Kozlov and V. F. Ezhov, Phys. Rev. A 49, 4502 (1994).

[102] L. B. Knight, W. C. Easley, and W. Weltner, J. Chem. Phys. 54, 322 (1971).

[103] C. Ryzlewicz, H. U. Schütze-Pahlmann, J. Hoeft, and T. Törring, Chem. Phys. 71, 389 (1982).

[104] L. I. Schiff, Phys. Rev. 132, 2194 (1963).

[105] H. M. Quiney, J. K. Laerdahl, K. Faegri, Jr, and T. Saue, Phys. Rev. A 57, 920 (1998).

[106] V. A. Dzuba, V. V. Flambaum, J. S. M. Ginges, and M. G. Kozlov, Phys. Rev. A 66, 012111 (2002).

[107] N. S. Mosyagin, A. V. Titov, and Z. Latajka, Int. J. Quantum Chem. 63, 1107 (1997).

[108] U. Kaldor and E. Eliav, Adv. Quantum Chem. 31, 313 (1999).

[109] T. A. Isaev, N. S. Mosyagin, M. G. Kozlov, A. V. Titov, E. Eliav, and U. Kaldor, J. Phys. B 33, 5139 (2000).

[110] N. S. Mosyagin, E. Eliav, A. V. Titov, and U. Kaldor, J. Phys. B 33, 667 (2000).

[111] W. R. Johnson and G. Soff, At. Data Nucl. Data Tables 33, 406 (1985).

[112] G. Fricke, C. Bernhardt, K. Heilig, L. A. Schaller, L. Schellenberg, E. B. Shera, and C. W. Dejager, At. Data Nucl. Data Tables 60, 177 (1995).

[113] D. Egorov, J. D. Weinstein, D. Patterson, B. Friedrich, and J. M. Doyle, Phys. Rev. A 63, 030501(R)/1 (2001).

[114] R. J. Buenker and S. D. Peyerimhoff, Theor. Chim. Acta 35, 33 (1974).

[115] F. Martin, R. Bacis, J. Verges, J. Bachar, and S. Rosenwaks, Spectrochim. Acta 44A, 889 (1988).

[116] K. P. Huber and G. Herzberg, Constants of Diatomic Molecules (Van Nostrand-Reinhold, New York, 1979).

[117] L. R. Hunter, S. E. Maxwell, K. A. Ulmer, N. D. Charney, S. K. Peck, D. Krause, S. Ter-Avetisyan, and D. P. DeMille, Phys. Rev. A 65, 030501 (2002).

[118] D. Kawall, Y. Gurevich, and D. DeMille, to be published. 